\title{
RECURRENT TRANSITORY ISCHEMIC ATTACKS WITH SUBSEQUENT DEVELOPMENT OF THE ISCHEMIC STROKE AS INITIAL MANIFESTATION OF EOSINOPHILIC GRANULOMATOSIS WITH POLYANGIITIS IN DEPRESSED PATIENT
}

\author{
Darija Mahovic $^{1}$, Nenad Lakusic ${ }^{2}$, Ivana Ljubicic ${ }^{3}$, Nadica Laktasic Zerjavic ${ }^{1}$, \\ Katarina Ivana Tudor $^{1} \&$ Gordan Pavlisa ${ }^{1}$ \\ ${ }^{1}$ University Hospital Center Zagreb, School of Medicine Zagreb, University of Zagreb, Zagreb, Croatia \\ ${ }^{2}$ Hospital for Medical Rehabilitation Krapinske Toplice, Faculty of Medicine Osijek, \\ Josip Juraj Strossmayer University of Osijek, Krapinske Toplice, Croatia \\ ${ }^{3}$ University Hospital Center Rijeka, School of Medicine Rijeka, University of Rijeka, Rijeka, Croatia
}

received: 28.11.2016;

revised: 19.4.2017;

accepted: 1.6.2017

\section{INTRODUCTION}

Eosinophilic granulomatosis with poliangiitis (EGPA; formerly known as Churg Strauss syndrome) is a systemic disorder characterised by asthma, peripheral eosinophilia, sinusitis and affection of different organic systems, e.g. peripheral nervous system, skin, kidney and gastrointestinal tract. EGPA is a rare entity that is characterised by widespread vasculitis which affects both small and medium - sized blood vessels, but in most cases initial clinical manifestations are asthma and skin changes (Sinico \& Bottero 2009). The pathogenetic mechanisms are not completely clear. Following criteria are widely used for the EGPA diagnosis: asthma, eosinophilia (more than $10 \%$ in complete blood count CBC), mono- or polyneuropathy, pulmonary infiltrates (may be transient), paranasal sinusitis and extra-vascular eosinophils (biopsy of arteries, arterioles or venules may demonstrate presence of eosinophilic infiltrates in the extravascular area) (Groh et al. 2015). EGPA is a rare systemic disorder. The annual incidence varies according to the published literature, with estimated prevalence of 17.8/ 1.000.000 (Watts et al. 2005). The most common neurologic manifestation of EGPA are peripheral neuropathy, usually mononeuritis simplex which untreated, may progress to symmetric or asymmetric polyneuropathy. Central nervous system (CNS) involvement (cerebral infarction and haemorrhage) is rare (Wolf et al. 2010). Eosinophilic vasculitis affects central nervous system (CNS) in less than $10 \%$ of cases (Sinico \& Bottero 2009). We present a case of a male patient with EGPA and depression that initially manifested with atypical and rare clinical presentation of recurrent transitory ischemic attacks (TIAs) with subsequent development of the ischemic stroke.

\section{CASE REPORT}

61-year-old patient with history of depression, arterial hypertension and chronic sinusitis presented with sudden onset weakness of the left extremities. After careful examination at emergency room patient was hospitalised in the neurology department. Complete regression of neurological deficit occurred an hour after the onset of neurological symptoms. On admission to the hospital, the patient has given his written informed consent for all necessary diagnostic procedures and treatment. Multi Slice Computed Tomography (MSCT) of the brain that was performed in emergency department prior to admission did not detect ischemic, haemorrhagic stroke nor intracranial mass lesions. Two weeks prior to the hospitalisation, patient had a similar attack of sudden left-sided weakness, which lasted approximately ten minutes, with complete recovery for reasons he did not seek medical assistance. Besides current neurological symptomatology, patient complained of fatigue, irritative cough, loss of appetite and weight loss, weakness in lower legs, feet and hand muscles with intermittently sub-febrile temperatures that started few months earlier. Because of these symptoms the patient was examined at a local hospital few months before, and aditional pulmonary evaluation was recommended, which was not done before the hospitalisation. Patient was examined by a psychiatrist and depressive episode was diagnosed and treatment with escitalopram started, which was gradually titrated up to $20 \mathrm{mg}$. Neurological examination revealed hoarseness, hypotrophy of the lower legs and feet with hypotrophy of the hand muscles (more prominent on the right), with diminished myotatic reflexes. Patient was mobile but had a wide - based gait and was incapable to stand on heel and toes. Laboratory testing found increased Creactive protein (CRP $88 \mathrm{mg} / \mathrm{L}$ ), erythrocyte sedimentation rate (ESR $36 \mathrm{~mm} / \mathrm{h}$ ), leukocytes (L $19.3 \times 109 / \mathrm{L})$, eosinophilia (57\%), slight normocytic anaemia (Hemoglobin - Hb $115 \mathrm{~g} / \mathrm{L}$ ) and mild dyslipidaemia (Low density lipoprotein - LDL - cholesterol $3.8 \mathrm{mmol} / \mathrm{l})$. Coagulation tests were in normal value ranges.

Patient was initially treated with a acetylsalicylic acid (300 mg first day, then $100 \mathrm{mg}$ per day) and 
atorvastatin $(20 \mathrm{mg})$. Color Doppler Flow Imaging (CDFI) excluded significant stenosis of carotid arteries. Echocardiography demonstrated normal systolic function without enlargement of the ventricles and valvular system without significant dysfunction. 24-hour Holter electrocardiogam showed stabile sinus rhythm, without significant ventricular ectopic activity and paroxysmal atrial fibrillation. On the third day of hospitalisation, left-sided weakness occurred again with left-sided supranuclear lesion of the facial nerve that persisted throughout the duration of the hospitalisation. Few hours later a magnetic resonance imaging (MRI) of brain was ordered and cerebrospinal fluid (CSF) was checked. MRI demonstrated an acute ischemic lesion in the area of the right thalamus and posterior limb of internal capsule with multiple, bilateral, postischemic lesions in basal ganglia and deep white matter, more pronounced on the right side. MR-angiography demonstrated diffuse irregularities of cerebral arteries and stenosis of M1 and M2 segment of right middle cerebral artery (MCA) and stenosis of distal M1 segment of left MCA (Figure 1). Electromyoneurography (EMNG) showed polyneuropathy. History of chronic sinusitis and cough prompted us to do more detailed investigations of respiratory system. Chest $\mathrm{x}$-ray did not reveal infiltrates or signs of cardiac failure. Spirometry detected moderate obstructive disturbances of ventilation on the level of small respiratory airways. Bronchodilator reversibility testing with salbutamol was positive. Due to repeated eosinophilia, immunological and stool analysis were performed, that were negative, while haematological investigations excluded primary hypereosinophilic syndrome. CSF analysis showed normal cell count with increased proteins and identical IgG bands in CSF and serum (intrathecal synthesis excluded). Immunological investigations demonstrated positive Perinuclear Anti-Neutrophil Cytoplasmic Antibodies - $p$ - ANCAs $(1: 320$, normal value < 1:20) and rheumatoid factor - RF (53.7 IU/mL, normal value $<14 \mathrm{IU} / \mathrm{ml})$. With regards to the clinical course and above mentioned results of the extensive diagnostic evaluation, we made the diagnosis of the EGPA. Treatment with methylprednisolone $500 \mathrm{mg}$ per day 8 for 5 days and Cyclophosphamide was initiated what led to partial recovery in patient status. Patient was referred in rehabilitation institution for further rehabilitation treatment. After completion of rehabilitation, control examination was performed three months after initial hospitalisation. Patient condition was stable with mild left - sided weakness residue and without a prominent cough. Further controls by immunologist - rheumatologist were advised.

\section{DISCUSSION}

EGPA is the most infrequent of the ANCA vasculitides. The exact pathogenetic mechanism of this disorder is unclear. pANCA are present in about half of the affected patients (Groh et al. 2015, Comarmond et al. 2013). It is not known whether pANCAs have a pathogenetic role in EGPA or they just reflect one end of the spectrum of EGPA manifestations. Patients with positive antibodies usually have renal and CNS involvement, while seronegative patients more often have cardiac manifestations (Sinico \& Bottero 2009). In 75\% of the patients peripheral nervous system is affected (polyneuropathy or mononeuritis multiplex), while CNS involvement is extremely rare and according to literature data it is less than 10\% (Watts et al. 2005, Wolf et al. 2010).

We report a case of 61-year-old male patient with history of depression who presented with two TIAs and subsequent development of the ischemic stroke in the region of the MCA. Even though patient had risk factors for the cerebrovascular disorders (arterial hypertension and mild dyslipidaemia), we made a diagnosis of EGPA based on a clinical and laboratory signs that included chronic sinusitis, eosinophilia, polyneuropathy and asthma, as well as presence of p-ANCAs. Furthermore, depression significantly increased the risk and develoment of stroke and this increase is probably independent of other risk factors (Dong et. al. 2012). In the published literature, we found several cases of EGPA where patients suffered from a cardioembolic stroke (Cheng et al. 2012, Ghaeni et al. 2010, Bhagirath et al. 2009). More than half of the patients with EGPA have cardiac involvement with eosinophilic infiltration that leads to endomyocardial fibrosis and overlying thrombosis and as a most common complication eosinophilic endocarditis (Sinico \& Bottero 2009). Cheng MJ et al. concluded that middle aged women with EGPA have a tendency for development of the subarachnoidal haemorrhage, while intracerebral haemorrhage (ICH) is more often present in men with EGPA that are middleaged or younger (Cheng et al. 2012). Mencacci et al. presented the 29-year old male patient with $\mathrm{ICH}$ as a first manifestation of the EGPA (Mencacci et al. 2011). In regards to normal cardiological, as well as, neuroradiological investigations in our patient, it can be assumed that recurrent TIAs, with subsequent development of the ischemic stroke are a result of the CNS vasculitis. Systemic vasculitis may cause damage of the walls of cerebral blood vessels in varying degrees (stenosis, aneurysms or occlusions) resulting in ischemia or haemorrhage (Sinico \& Bottero 2009). The MRangiography in this case revealed diffuse irregularities of cerebral arteries and stenosis of M1 and M2 segment of right MCA and stenosis of distal M1 segment of left MCA. Primary therapy of EGPA is systemic glucocorticoids. Immunosuppressants such as cyclophosphamide are also required, in patients with CNS involvement and pANCAs presence (Sinico \& Bottero 2009, Mukhtyar et al. 2009). The patient's general condition improved on steroid and immunosuppressive therapy, but he is still in need of neurological support and rehabilitation. 


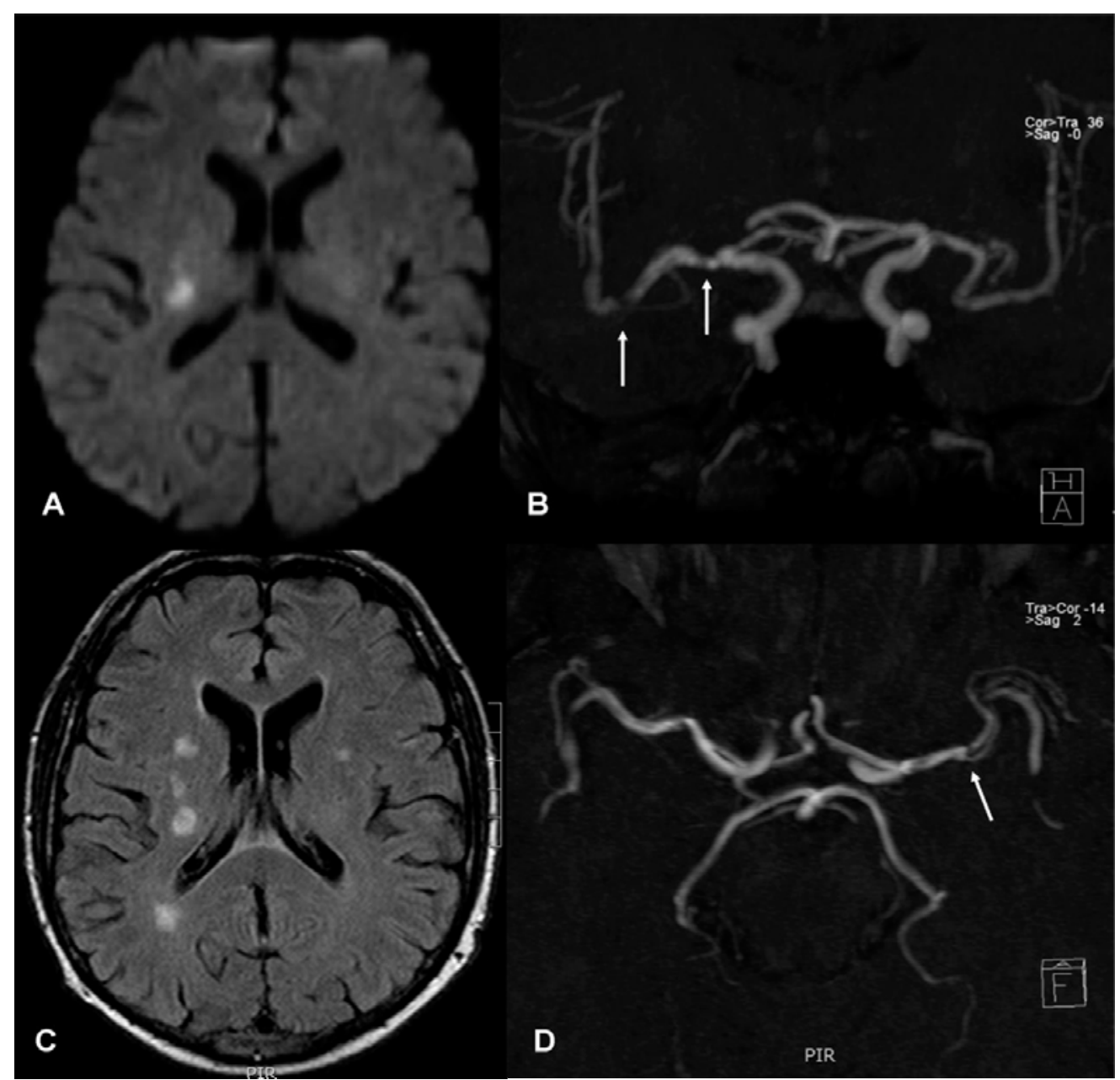

A: Diffusion-weighted MR image showing an acute ischemic lesion at the level of right thalamus and posterior limb of internal capsule. B: 3D time-of-flight MR angiography in coronal plane showing diffuse irregularities of cerebral arteries and stenosis of M1 and M2 segment of right middle cerebral artery (arrows). C: Fluid-attenuated inversion recovery image in axial plane reveals multiple postischaemic lesions in basal ganglia and deep white matter, more pronounced on the right side. D: 3D time-of-flight MR angiography in axial plane. Arrow is pointing a stenosis of distal M1 segment of left middle cerebral artery.

Figure 1. Brain MRI and MRI angiography

In published literature we did not find reports of EGPA and TIA. EGPA is the rarest of the antineutrophilic cytoplasmic antibody vasculitides. In this case, occurrence of TIA in our patient, was the first, severe symptoms and initial manifestation of EGPA and reason for hospitalisation.

\section{CONCLUSION}

Although, patient had a history of chronic sinusitis, weight loss, subfebrility, fatigue, chronic cough, paraesthesia and muscle pain, recurrent TIAs with subsequent development of the ischemic stroke were the initial, important manifestation that resulted in the hospitalisation and detailed diagnostic procedures. We would like to emphasise, that when managing patients with TIAs or stroke, besides more common risk factors such as atherothrombosis or thromboembolism as a result of atrial fibrillation, it is also important to consider other, rare causes that may require specific treatment.
Acknowledgements: None.

Conflict of interest: None to declare.

\section{Contribution of individual authors:}

Darija Mahovic: design of the case report, first draft, literature searches and analyses, approval of the final version;

Nenad Lakusic: design of the case report, first draft, literature searches and analyses;

Ivana Ljubicic: first draft, literature searches and analyses;

Nadica Laktasic Zerjavic: literature searches and analyses;

Katarina Ivana Tudor: firt draft, literature searches and analyses;

Gordan Pavlisa: radiology diagnosis and interpretation of the images, approval of the final version. 


\section{References}

1. Bhagirath KM, Paulson K, Ahmadie R, Bhalla RS, Robinson D, Jassal DS. Clinical utility of cardiac magnetic resonance imaging in Churg-Strauss syndrome: case report and review of the literature. Rheumatol Int. 2009; 29:445-9.

2. Cheng MJ, Huang PH, Liao PW, Chen JT, Chiang TR. Multiple cerebral and cerebellar infarcts as the first clinical manifestation in a patient with Churg-Strauss syndrome: case report and literature review. Acta Neurol Taiwan. 2012; 21:169-75.

3. Comarmond C, Pagnoux C, Khellaf M, Cordier JF, Hamidou $M$, Viallard JF, et al. Eosinophilic granulomatosis with polyangiitis (Churg-Strauss): clinical characteristics and long-term followup of the 383 patients enrolled in the French Vasculitis Study Group cohort. Arthritis Rheum. 2013; 65:270-81.

4. Dong Jia-Yi, Zhang Yong-Hong, Tong Jian, Qin Li-Qiang. Depession and Risk of Stroke. A Meta - Analysis of Prospective Studies. Stroke 2012; 43:32-37.

5. Ghaeni L, Siebert E, Ostendorf F, Endres M, Reuter U. Multiple cerebral infarctions in a patient with ChurgStrauss syndrome. J Neurol. 2010; 257: 678-80.
6. Groh M, Pagnoux C, Baldini C, Bel E, Bottero P, Cottin $V$. et al. Eosinophilic granulomatosis with polyangiitis (Churg-Strauss) (EGPA) Consensus Task Force recommendations for evaluation and management. Eur $J$ Intern Med. 2015; 26: 545-53.

7. Mencacci NE, Bersano A, Cinnante CM, Ciammola A, Corti $S$, Meroni PL, et al. Intracerebral haemorrhage, a possible presentation in Churg-Strauss syndrome: case report and review of the literature. J Neurol Sci 2011; 3011: 107-111.

8. Mukhtyar C, Guillevin L, Cid MC, Dasgupta B, de Groot $K$, Gross W, et al., for the Europe- an Vasculitis Study Group EULAR recommendations for the management of primary small and medium vessel vasculitis. Ann Rheum Dis. 2009; 68: 310-17.

9. Sinico RA, Bottero P. Churg-Strauss angiitis. Best Pract Res Clin Rheumatol. 2009; 23: 355- 66.

10. Watts RA, Lane S, Scott DG. What is known about the epidemiology of the vasculitides? Best Pract Res Clin Rheumatol. 2005; 19: 191-207.

11. Wolf J, Bergner $R$, Mutallib S, Buggle F, Grau AJ. Neurologic complications of Churg-Strauss syndrome--a prospective monocentric study. Eur J Neurol. 2010; 17: 582-8.

Correspondence:

Prof. Darija Mahovic, MD, PhD

University Hospital Center, Zagreb, School of Medicine Zagreb, University of Zagreb

Kišpatićeva 12, 10000 Zagreb, Croatia

E-mail: darija.mahovic@zg.t-com.hr 